\title{
BIOMARS: A Foundational High-Resolution Environmental Sensor Array
}

\author{
A Mission Concept White Paper submitted to the Planetary Science and Astrobiology \\ Decadal Survey 2023-2032
}

\section{Lead Author:}

Nathalie A. Cabrol, SETI Institute Carl Sagan Center, ncabrol@seti.org

\begin{abstract}
Co-Authors
Lori K. Fenton, SETI Institute, 1fenton@seti.org; Kimberley Warren-Rhodes, The SETI Institute, kim_lamma@yahoo.com; John Hines, Hines Family Foundation, johnhines555@gmail.com; Nancy Hinman, University of Montana, nancy.hinman@mso.umt.edu; Jeffrey Moersch, University of Tennessee, Knoxville, imoersch@utk.edu; Pablo Sobron, SETI Institute, psobron@seti.org; David S. Wettergreen, CMU-RI, Pittsburgh, dsw@ri.cmu.edu; Kris Zacny, Honeybee Robotics, KAZacny@honeybeerobotics.com; Margaret Race, SETI Institute, mrace@seti.org; Jesus Martinez-Frias, IGEO (CSIC-UCM), Madrid, Spain, j.m.frias@igeo.ucmcsic.es; Flora Paganelli, paganelliflora@gmail.com; Jen Blank, Blue Marble Institute/NASA Ames, jen@bmsis.org;
\end{abstract}

Co-Signers: Geoffrey Landis, NASA Goddard Space Flight Center, geoffrey.landis@nasa.gov; Alberto Fairen, CAB-INTA, Spain and Cornell University, agfairen@cab.inta-csic.es; Dale Andersen, SETI Institute, dandersen@seti.org; Janice Bishop, SETI Institute, jbishop@seti.org; Richard Cartwright, SETI Institute; rcartwright@seti.org; Anthony Dobrovolskis, SETI Institute; adobrovolskis@seti.org; Mark Elowitz, (SETI Institute Affiliate), elowitzm@gmail.com; Inge Loes, Universiteit Utrecht, I.L.tenKate@uu.nl; Carl Shneider, CWI, The Netherlands, Carl.Shneider@cwi.nl; Virginia Gulick, SETI Institute, vgulick@seti.org; Doug Caldwell, SETI Institute, dcaldwell@seti.org; Bill Diamond, SETI Institute, bdiamond@seti.org; Timothy Michaels, SETI Institute, tmichaels@seti.org

All authors have given permission to be named on this document 


\section{Overview}

Whether to augment the current robotic program of biosignature exploration, support future human colonization and astrobiological investigations [1], or provide key data to understand Earthderived contamination, environmental data on Mars are critically important. Mars orbiters produce datasets that support current global to mesoscale climate models. InSight acquires environmental information but only at one site, and its focus is mainly on seismic data. Rovers collect daily environmental data at discrete locations (e.g., REM, MEDA). However, rovers are mobile platforms with instrument packages that are heterogeneous from one mission to the next, and data are rarely acquired at the same site daily, making interpretation more complex. Pathfinder has shown that substantial temperature differences can be induced by meter-scale topographic heterogeneities. These may affect weather locally and regionally in ways that still need to be quantified and understood, particularly when thinking about scales relevant to microbial habitats. While no life is expected to survive at the surface of Mars today, the study of terrestrial extreme environments shows that subaerial hotspots can provide indications of subsurface to deeper surface microbial oases. Therefore, understanding environmental variability at a resolution relevant to microbial habitats ( $\mathrm{mm}$ to $\mathrm{nm}$ scales) is a critical, and currently strategically missing element, in the planning of Mars missions.

BIOMARS calls for the deployment of a large number of small sensor nodes, over vast areas of Mars' surface to enable the collection of high-resolution environmental data. Such a mission would provide key datasets that could give a profound insight into Mars past and present astrobiological potential and help prepare for its future exploration and colonization.

\section{A Strategic Mission Gap}

Mars missions have gathered information about the nature and state of the atmosphere from orbit. Past rovers, now Curiosity, soon Perseverance, Rosalind Franklin, and the Chinese rover will collect sedimentologic and environmental data as they move along traverses on the ground. Rovers also acquire atmospheric data, including combined observations of the atmospheric column with orbiters. However, much of the knowledge that will be critical to upcoming [e.g., 2-3] and future exploration, whether astrobiological or human, is still lacking. Orbital data provide limited information about the lowest few kilometers of the atmosphere, and spatial resolution is seldom sufficient to resolve kilometer-scale or finer atmospheric processes. In contrast, Mars rovers collect daily environmental datasets, but rovers are mobile platforms, and resulting datasets are difficult to compare. Further, the meteorological datasets acquired thus far by landed spacecraft have been limited by calibration issues, poor or intermittent sampling, low instrument sensitivity, and both mechanical and thermal contamination from the spacecraft themselves.

Missing from past, current, and planned landed missions on Mars is a suite of instruments that work synergistically to fully characterize near-surface environment and the surface/atmosphere interactions through long-term ( $>1$ Martian year) monitoring of atmospheric and soil/regolith conditions. This can be accomplished through measurements of parameters including air and surface temperature, air pressure, relative humidity, wind velocity, dust loading, trace gas concentration, soil $p \mathrm{H}$, and other environmental variables. Notably, this includes high-frequency measurements that enable quantification of turbulent fluxes governing the exchange of heat, momentum, volatiles, and dust between the surface and atmosphere. These parameters characterizing the local microclimate are essential to (a) quantify the preservation potential of ancient organic biomarkers and to (b) identify unique conditions for present-day near surface 
microbial habitat potential. They influence larger atmospheric circulations that drive both the broader Martian climate system and the mesoscale conditions needed to safely conduct entry, descent, and landing operations.

The need for high fidelity surface meteorology data is well-recognized in the Mars science community. For example, a white paper by Rafkin et al. [4] stated that "the next major increase in our understanding of the near-surface environment will come from high quality systematic measurements of winds. For this reason, winds are the next higher priority for any landed meteorology payload after pressure and temperature. Surface fluxes are major forcing functions of atmospheric motions, yet very little is known about their magnitude and variability". Further, it has been recognized that a long-lived network of environmental instruments is critical to characterizing the variability and diversity of meteorological conditions on Mars [e.g., 4-6].

To fill this gap, we recommend the inclusion in the Mars Exploration Program (MEP) of strategic planning for the design, development, and deployment of a mission concept to characterize and quantify near-surface environmental variability on Mars at high spatiotemporal resolution. An instrument suite with these capabilities will address numerous MEPAG objectives and high-priority investigations, including those that call for detailed measurements of atmospheric and surface conditions, biosignature preservation potential, and environmental knowledge required for designing and implementing human missions [6].

\section{Science Goals and Objectives}

In the search for biosignatures, early and present Mars must be conceptualized as a biosphere, and exploration should vigorously integrate an ecosystem approach to missions - an element that is direly missing today [7]. While life as we know it cannot survive directly exposed at the surface today, research in terrestrial extreme environments shows that habitats can still develop near the surface. Their evolution in such environments is predominantly dependent on local, not global factors, and highly influenced significantly by the landscape (e.g., topography, geology, texture, albedo), and its variability at a microbial habitat scale. Such habitats are characterized by unique conditions at multiple scales, but particularly at the meter to micrometer scale (e.g., slope exposure, cracks in rocks, cavities in ancient lakebed muds, surface/atmosphere interactions such as deliquescence, UV radiation shielding, other), e.g., [8-9]. At individual sites, parameters such as temperature, moisture, light, mineralogy, sediment texture, $p \mathrm{H}$, and others, generally differ from the average regional ambient environment, and remain stable over time, in order for life to persist.

Further, the conditions conducive to the formation of microbial habitats, the frequency at which they fluctuate, and how they evolve over time, are dictated by local factors such as energy sources, mineralogy, geochemistry of sediments, their texture, water acquisition, moisture retention; energy balance between the habitat and the atmosphere, and shielding (e.g., from UV). They are also driven by the interactions between multiple environmental factors and their relative dominance in time. At larger scale, this would involve the interplay of polyextreme environmental factors on Mars [10], but the same is true at the microscale, where local environmental conditions sustaining microbial habitats interact in an ecosystem with complex loops and feedback mechanisms. There are currently no datasets available to start conceptualizing the nature of such interplays on Mars, nor an understanding of the types of putative microbial habitats they could sustain (or may have sustained in the past). Such datasets would allow us to model microbial response (e.g., adaptation, metabolism, genetic) to the interplay of these environmental extreme factors, and begin 
envisioning the plausible bio/geosignatures they could leave behind (e.g., biomediated minerals, pigments, morphologies, textures).

High-resolution environmental data would also bring important information about surface erosion and the preservation of such bio/geosignatures. Surface erosion by windblown materials has likely been the dominant mechanism exposing any potential remains of biological materials in the past 3 billion years. However, the physics of particle transport and surface abrasion on Mars are not well understood, and current saltation and atmospheric models do not explain the observed behavior of mobilized particles.

Surface environmental datasets have critical value for the strategic planning of the Mars exploration and for considerations of planetary protection. For instance, microbes and habitats are impacted differently by sudden surface exposure or radiation accumulation doses [e.g., 11]. Exposure is rapidly lethal for microbial organisms, although temporary survival may be possible under thin layers of ice, dust or regolith [12]. Recent results at Gale crater show that the ionizing radiation flux reaching the surface is constant and negligible [13]. Biomolecules are compromised by dose accumulation at a rate of $10 \mathrm{~cm}$ depth per 300 million years [14], not instant exposure. Sterilization depth may also vary depending on the mineralogical composition and structure of the subsurface [15-16].

\section{A High-Resolution Environmental Sensor Arrays on Mars}

High-resolution environmental arrays deployed strategically, would map the distribution and concentration of astrobiological hotspots dynamically. They could become a cornerstone strategy for the exploration of Mars, and support four key exploration goals:

(A) Support the search for biosignatures;

(B) Provide ground-truth measurements to atmospheric global circulation models;

(C) Foster a better understanding of planetary protection requirements through the identification and characterization of environmental hotspots below current resolution, and their relationships with respect to present and future missions; and

(D) Prepare and support the human exploration of Mars.

Data from such arrays would be critical to: (a) site selection prior to the deployment of a human crew or a habitat site, (b) weather forecasting at colony sites (e.g., dust storms, dust devil formation potential, magnitude, frequency, atmosphere dynamics, particle transport, radiation environment); (c) human habitat safety and maintenance (e.g., EVA planning) where particle size and shape considerations are critical to characterize dust penetration potential in spacesuit and breathing apparatus. They would also contribute to: (i) scientific activities, including astrobiological exploration, where local environmental data will provide critical background to put findings into context; (ii) local resources (e.g., understanding of regolith properties for cultures, such as grainsize and shape for water circulation and retention potential, soil $p \mathrm{H}$, other); and (iii) prevention of contamination. Some terrestrial organisms can survive UV radiation for hours or longer, and windblown transport from a crewed habitat to sheltered areas in the Martian soil could result in contamination, where such organisms could metabolize and possibly replicate. To avoid forward contamination via wind, main paths of dispersal must be determined.

Scientifically, a high-resolution characterization of the scales of variability of the Martian environment over a range of relevant spatial scales is an exciting and urgently needed mission concept that can be brought on the path to launch within the next decade. Such a mission would 
involve strong public/private partnerships with industry for technology development and advanced data analytics. Instruments and systems will benefit from the current Internet of Things (IoT) revolution in the monitoring of the terrestrial environment and foster the design, development, and testing of intelligent communication systems. These systems will be supported by Artificial Intelligence (AI) at all levels, including acquisition, processing, transfer, and analysis of the vast amount of data generated by such missions. Critically, the concept can be fully tested on Earth through precursor simulation missions in extreme environments, a key outcome being the development of novel ways of monitoring our own changing planet and the evolution of microbial habitats and their diversity, which are the linchpins of terrestrial biogeochemical cycles, undergird all ecosystem services to both the natural and human world, and are a central component in sustaining the health and function of all macro-organisms on our planet [17-18].

\subsection{Mission Concept}

Sensor Arrays can be deployed either as a cluster of several modules (defining a node) in close proximity of each other at one site; or as several sets of clusters deployed in various environments, where landing locations are dictated by mission and modeling goals (e.g., latitudes, topography, geology, mineralogy, texture/albedo). A key minimum requirement for a local cluster suite would include the geographic proximity of modules $(\leq 1 \mathrm{~km})$ with variations in topography, slope exposure, or any other factors that may generate distinct local conditions. The baseline mission would be to collect data over a minimum of a Martian year. Environmental data have low bandwidth requirements for transmission and therefore, a high-resolution data sampling rate could be achieved over long periods for multiple datasets at multiple fixed locations.

Development and testing areas for such a mission concept could include, but are not limited to, the design of individual communication systems; onboard data acquisition and onboard processing, analysis, and data transfer. Environmental detection algorithms developed for planetary simulations [e.g.,19-21] and actual missions [22-23] can be improved and upgraded. These algorithms, together with novel AI tools would be applied onboard and/or off-board to datasets to execute pattern detection, identification of anomalies, event detection for the atmosphere (clouds, dust devil, other) and surface-near-subsurface-atmosphere interactions. New deployment and exploration methods, instrument payload packaging and systems need to be designed, developed, and field tested with a high level of environmental analog fidelity. While there is no perfect analog to Mars on Earth, high altitude/low latitude extreme environments present many of the conditions that will enable the development of baseline mission requirements, including thin, unstable atmosphere, seasonal dust devil and dust storm occurrence, aridity, high daily and seasonal temperature variability, UV radiation - including short UV, soil geochemistry, and overall geology, and high rate of evaporation. Integrated field tests in such environments would also help determine optimal sampling rates and complete mission preparation.

\subsection{Example Payload}

The main goal of BIOMARS is acquisition of knowledge of environmental processes relating to atmospheric circulation, atmosphere/surface/near subsurface interactions, microbial habitats, biological activity, and biomarker preservation. Instrument accommodation should be designed to maximize high fidelity data return, and to address these scientific concerns they should be deployed to avoid physical or thermal interference from the lander body. An instrument suite should include, but is not limited to: 
Mission Floor Gradient Instruments: Pressure Sensor at $\geq 1 \mathrm{~Hz}$ would record variations in $\mathrm{CO}_{2}$ abundance, ranging from seasonal to diurnal oscillations (e.g., thermal tides) to those produced by mesoscale (e.g., baroclinic waves), topographic, and convective processes (e.g., dust devils). Anemometer capable of measuring wind velocities in 3 dimensions and air temperature, with a frequency $\geq 10 \mathrm{~Hz}$ required to measure all fluxes, including turbulent momentum and sensible heat fluxes. The instrument package would provide diurnal and seasonal wind, and temperature series. A Relative Humidity sensor $\geq 1 \mathrm{~Hz}$ would be required to obtain moisture flux in both directions (when combined with acoustic anemometer wind velocities) at the air-regolith interface.

Mission Baseline Instruments - In addition to above Floor, the baseline mission would include: Dust sensor at $0.1 \mathrm{~Hz}$, measuring suspended dust concentrations, size distribution, speed and deposition rate. It would provide temporal variation in dust grain size and concentration variation with flows caused by diurnal, seasonal, convective, mesoscale-related flows. In combination with the anemometer, it would provide dust fluxes and wind velocity threshold for dust entrainment. A Saltation sensor would measure speed, mass, and flux of saltating sand grains. In combination with the anemometer, it would provide wind velocity threshold values for sand saltation, and an estimate of surface abrasion rates. Methane and $\mathrm{CO}_{2}$ sensors would identify gas concentration and flux. In combination with the anemometer, directions to sources could be identified. Visible/Multispectral Imager(s) document the surrounding terrain (e.g., rock abundance/distribution, bedforms, sediment texture, composition, soil grain-size distribution), transient event (e.g., saltating particles, clouds, dust devils), and optical depth of ice and dust aerosols through direct solar imaging.

Beyond Baseline - (Includes above baseline, and in priority order): An Outgoing (downward looking) Longwave Radiation Sensor, radiative forcing term to close energy budget; Incoming (upward looking) longwave radiation sensor, radiative forcing term to close energy budget. Incoming (upward looking) shortwave radiation (insolation) sensor, radiative forcing term to close energy budget. It can also be used to measure atmospheric optical depth (redundancy with dust sensor) and descent profiles of air density or temperature. This would provide a rare opportunity for atmospheric structure measurement - considered a medium priority PSAG Gap-Filling Activity. Soil moisture, to determine ground heat flux and thermal inertia. Lidar to provide vertical profile of aerosols (dust and water ice). This is consistent with a high-priority MEPAG investigation and PSAG Gap-Filling Activity.

\section{Surface Delivery}

The small individual size of the nodes $(10-20 \mathrm{~cm})$ allows to deliver them in various ways depending the envisioned distribution (global, regional, local). For larger spray (global and regional) at the surface of Mars, they can be jettisoned during descent into the martian atmosphere. For local delivery of clusters of nodes, they can be carried and deployed by a rover. The size of the BIOMARS array can be incrementally increased over time with additional nodes piggybacking on other missions. Landing additional sets of modules to cover key regions of Mars that include basic communication assets could be used as a starting ground communication system for future human exploration.

\section{Conclusion}

SMD Astrobiology programs such as PICASSO and MATISSE could be used to advance instrument development and integration forward; the PSTAR program could field test integrated 
systems, investigation methods, and science operations. Industry partners could contribute AI research and development alongside NASA and academic institutions, and off-the-shelf instrument components or systems developed for environmental monitoring.

From a science standpoint, such a mission would fill a critical knowledge gap. Although our understanding of early Mars in its first billion of years remains fragmented, present-day Mars is a reflection of its integrated past 3 billion years of history. Therefore, a characterization of the highresolution spatiotemporal scale of variability of the environment today, combined with our understanding of obliquity cycles and climate forcing, will provide an immediate, and uncertaintyfree insight into the formation of potential astrobiological hotspots at Mars' surface and nearsubsurface, and an end-member for an ecosystem development potential over 75 percent of its history.

Finally, the scientific and technological knowledge gained by this mission concept would reach across NASA Planetary, Astrobiology, Earth Sciences, and Human Exploration Divisions, providing key information to (a) support future Mars missions and build critical bridges between global, mesoscale atmospheric modeling and local weather; (b) provide in-depth knowledge of the environmental variability at a scale that matters for microbial habitats; (c) potentially reveal new information to develop a better understanding on how to approach exploration and planetary protection; (d) develop the technology and systems for high-performance planetary environmental surveys that can be applied to monitor more efficiently, and in novel ways, our own changing planet, and (e) provide the nucleus of a ground communication and weather forecasting system for future humans on Mars.

\section{References}

1. Beaty, D. W., et al., 2015. AGU Fall Meeting, December 2015; 2. Williford et al., 2018, In: From Habitability to Life, (Cabrol, N.A., and E. A. Grin, Eds), Elsevier, 390 pp; 3. Vago et al., 2017, ESA Bull. 126:16-23; 4. Mischna et al., 2009, NRC Decadal Survey, 2011-2020. http://mepag.nasa.gov/reports.cfm; 5. Rafkin et al., 2009, NRC Decadal Survey, 2011-2020, http://mepag.nasa.gov/reports.cfm; 6. MEPAG, 2015, http://mepag.nasa.go/reports/cfm; 7. Cabrol, 2018, Astrobiology, 18(1), doi: 10.1089/ast.2017.1756; 8. Cabrol et al., 2007a; J Geophys Res: Biogeosciences 112, doi:10.1029/2006JG000298; 9. Warren-Rhodes et al., 2007, J Geophys Res: Biogeo.112, doi:10.1029/2006JG000283; 10. Cabrol et al., 2007b, Proc SPIE 6694. doi:10.1117/12.731506; 11. Beaty et al., 2006, http://mepag.jpl.nasa.gov/reports/index.html.; 12. Rummel et al., 2014, Astrobiology 14:887-968; 13. Hassler et al., 2014, Science 343, doi:10.1126/science.1244797; 14. Pavlov et al., 2012, Geophys Res Lett 39, doi:10.1029/2012GL052166; 15. Gómez et al., 2010, Icarus 209:482-487; 16. Ertem et al., 2017, Int. J. Astrobiology, 16:282-285; 17. Rousk, J. and P. Bengston C., 2014. Front. Microbiol., doi.org/10.3389/fmic.2014.00103. 18. Hug, L. et al., 2016, Nature Microbiology 1: 16048. 19. Cabrol et al., 2014; 45th Lunar Planet. Sci. Conf., Abstract 1185; 20 Smith et al., 2014; 45th Lunar Planet. Sci. Conf., Abstract 1616; 21. Pedersen et al., 2015, J. Field Robot., 32(6): 860-879; 22. Castano, et al., 2008. M. Vis. Appl. 19 (5-6): 467-482; 23. Thompson 2007 IEEE Aerospace Conf., 10.1109/AERO.2007.352699. 